\title{
Human Cognition to Analyse Alcohol Use Disorder and Correlation with Internet Gaming Disorder
}

This paper was downloaded from TechRxiv (https://www.techrxiv.org).

\section{LICENSE}

CC BY 4.0

SUBMISSION DATE / POSTED DATE

$12-08-2020$ / 14-08-2020

CITATION

Pandey, Hari (2020): Human Cognition to Analyse Alcohol Use Disorder and Correlation with Internet Gaming Disorder. TechRxiv. Preprint. https://doi.org/10.36227/techrxiv.12786776.v1

DOI

10.36227/techrxiv.12786776.v1 


\section{Human Cognition to Analyse Alcohol Use Disorder and Correlation with Internet Gaming Disorder}

\author{
Hari Mohan Pandey \\ Department of Computer Science \\ Edge Hill University \\ St Helens Rd, Ormskirk L39 4QP Lancashire, UK \\ Pandeyh@edgehill.ac.uk
}

\author{
Abhishek Jain \\ Computer Science and Engineering \\ Amity School of Engineering and Technology, Amity Uniersity \\ Noida, India \\ j.avishek@gmail.com
}

\begin{abstract}
Playing games always been interest in child and adolescent. Technology specially internet given new dimension to gaming and over the decade gaming over internet attracted major percentage of player. Excessive gaming when not controlled also lead to the behavioral dysfunction along with physiological issues. World Health Organization (WHO) has added Internet Gaming Disorder (IGD) as potential mental disorder recently in Diagnostic and Statistical Manual for Mental Disorders (DSM-5) as research field to include in main manual. Research in this field gained interest, but about diagnostic criteria debate is still on in research group. For diagnosis of IGD, not only subjective symptoms but its underlying neurobiology needs to be included. Major behavioral addiction shares its symptoms with different addiction, which can be seen into IGD and Alcohol Use Disorder (AUD) also. In this paper, we aim to identify potential biomarkers to explore relationships between AUD and IGD symptoms using EEG signals recordings. Noise and other artifacts are removed during pre-processing and bands splitted in intermediate steps of proposed method. Spectral features are potential indicator in EEG data, for all five EEG bands power spectral density is computed and visible difference found in mean power on most of the channels for all bands. Across theta and beta bands, mean absolute power is greatly reduced in the frontal and prefrontal brain region. The results indicate symptoms are matching with impulsive behavior. Based on statistical analysis across we found similarities in EEG features in terms of emotional imbalance, high arousal, slow response inhibition and our study indicate Alcohol Use Disorder and IGD are correlated.
\end{abstract}

Keywords-Electroencephalogram, Alcohol Use Disorder, Internet Gaming Disorder, Spectral Features, PSD

\section{INTRODUCTION}

Millions of neurons are working under the covered cortex of human brain, which directly or indirectly controls thought, memory, action and behaviour. When neurons synaptic is active it generates a kind of electrical signal along with magnetic field. Child and adolescent mind are immature to discriminate the dependency development, whether it is about food or any habit or any activity. Over the years, technology has emerged in biomedical science. Several new and enhanced tools are available now a days for reading such activities. Electroencephalography (EEG) is one of the tools and it is widely used in different scale lab setup. Such recording is useful for analysis and to detect the functional or structural changes in the brain are developed due to any addiction or disorder. In past, traditional psychological methods of addiction diagnosis were only available, but these methods needed rich experience to identify and assess. Brain cells work together to send information in a form of signal from one brain region to another and responsible for normal physiology as well as behaviour inhibition. If any change due to neurodevelopmental, neuromechanical or addition cause the behavioural or physiological change occurs.

Neurological disorders, especially behavioural disorders are hard to ascertain with traditional psychological methods, used by psychologists, but it is crucial to discover them at early stage of a development to reduce the Global Burden of Disease (GBD) [2]. Neuroimaging help in diagnosing addiction of activity or substance up-to great extent. The term psychologist addiction is differentiated by few of the common symptoms like relapsing, loss of control, anxiety, depression, mood modification. Substance and behaviour addiction have higher prevalence rate. Alcohol use comes under the definition of substance use.

People with moderate and high consumption can be observed distinctly, where moderate use may come under social or cultural norm, on the other hand excess consumption can lead to binge dirking or disorder. Data indicates variation in alcohol consumption like lower amount in countries of North Africa and the Middle East, but very high consumption were recorded across Eastern Europe countries like Belarus, Russia, Czech Republic and Lithuania [1].

The world is changing at very fast pace, it has gone through different revolution. Convergence of digital media and platform has impacted a lot compared to others. Young adults and adolescent are majorly impacted though the use of digital platform. Gaming style and platform has changed from physical space to digital world. Gaming over internet is one of the interests in child and adolescents. Excessive gaming lead to the structural and functional changes in the brain areas which are involved in cognitive, reward and punishment feelings. As new generation is moving from the real-life social relations to online social sites, which slowly, if not controlled turn into online addiction.

World Health Organization (WHO) in their $11^{\text {th }}$ release of ICD has identified the need of research in the field of diagnosis and prevention of such disease, Internet Gaming Disorder (IGD) is one among them. Majority of player are attracted by MMORPGs and are played by great percentage of players, players connected virtually mostly through their social connection [3]. Recent growth was recorded to $290 \mathrm{M}$ USD in gaming industry and it is forecasting to become $1 \mathrm{~B}$ USD industry by 2021 [4].

A decade ago while there was less feasibility of biomedical instruments, self-reported psychological questionnaires-based assessment for internet addiction test was widely used. Young Internet Addiction Test which showed better result in Internet Addiction prevalence test used in [5-6]. Questionnaire specific to gaming were developed in recent past for psychometric assessment of IGD 
subjects [7]. Prevalence rate and severity is calculated by psychologist which majorly depends on expertise. It is one of the assessment methods, which does not need a big lab setup. Considerable variance of prevalence rate was observed in studies using questionnaires-based method for assessment [8], due to the difference in the use of selective samples of online surveys [9].

Item response theory (IRT) analysis came up with unidimensional paradigm for abuse and dependence criteria, and intermixture of the criteria across severity spectrum, defying the hierarchical rule of lesser severity of abuse [10]. One of the studies on social drinkers [11] highlighted the risk factors as impulsivity and alcohol subjective response in developing alcohol use disorder. Result of study also indicated as the alcohol concentration level increases subject is more responsive to questionnaires and feel positive mood. Also, sedation will continue in high impulsive subjects.

In another study [12] to identify the predictors of estimated blood alcohol concentration subjective response was examined. Final estimation of blood alcohol concentration was done based on stimulant effect during mid of drinking, which also one of the predictors for blackouts during drinking. Studies which use subjective methods are good indicator of concentration level and responsiveness of subjects, but it is not able to identify the alcohol use as disorder and functional, and structural changes in the brain due to disorder. Subjective methods which are available to diagnose and analyse behavioural symptoms are not enough to understand underlaying brain neurology. Researcher and clinicians need more objective method to overcome the issues with traditional methods.

Motivated by the aforementioned discussion on alcohol use disorder and correlation with Internet Gaming disorder, we here propose a novel instruments for assessing alcohol disorder and identifying a correlation with Internet Gaming disorder. In particular, the merits of the proposed LPTWSVM formulation are as follows:

- We present a comprehensive discussion on the exiting work to setup a demand of the proposed system.

- We presented our proposed model which utilizes EEG signals and human cognition for analysing alcohol use disorder (AUD) and identifying correlation with internet gaming disorder (IAD).

- We show rigorous simulation results to present the effectiveness of the proposed system.

A broad paper outline is given as follows: Section II gives the related work. Section III outlines the proposed model and simulation. Experimental results and discussions are given in Section IV. Finally, conclusions are drawn in Section V.

\section{Human Brain Structure And Eeg Signals}

A human's cognitive state can become apparent by the subjective experiences such as feeling, mood etc. Hence, measuring cognitive congruency in human would be beneficial to analyse human behaviour for certain activities based on person's mood.

Over the years there is a remarkable growth in neuroscience to understand the specific brain structure of human through electro-physiological methods such as EEG,
fMRI etc. It is noted that any conscious or unconscious activity that originates in the brain can be expressed in terms of generated electrical activities. These electro-physiological methods like EEG are highly temporal in nature, thus EEG signals can assist in providing a better understanding of participant's cognitive state at the time of observations. EEG signals measures voltage fluctuation that are generated due to ionic current flows within the brain neurons. This electrical activity is then used to understand that "how humans think". In general EEG signals are measured at various portions of scalp and typically ranges from 10-100 microvolt. Human brain is broadly divided into four main parts, namely, frontal, parietal, temporal and occipital as depicted in Figure 1 [28].

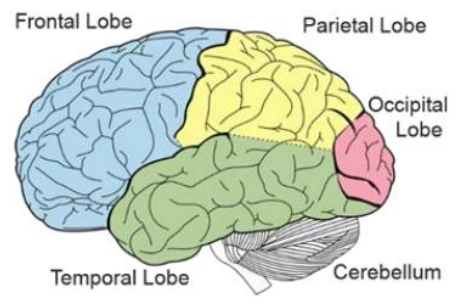

Fig.1. Human brain structure.

Each part is associated with processing of information differently. During visual information processing frontal lobe is usually related with working memory and decision related activities. Parietal lobe deals with the action related tasks and temporal lobe is involved in object recognition kind of tasks. Attention related information in processing visual information is executed through occipital lobe.

Human's cognitive state can be analysed using slow and high frequency ranges of the EEG waveform, i.e. Gamma (>30 Hs), Beta $(12-30 \mathrm{~Hz})$, Alpha $(8-12 \mathrm{~Hz})$, Theta $(4-8 \mathrm{~Hz})$ and Delta $(<4 \mathrm{~Hz})$. Slow waves have higher amplitude and lower frequency content as compared to high frequency bands. In certain cognitive state of mind, signals observed at scalp locations are prominent in certain frequency bands only. Thus, these frequency bands are usually used for examining the brain responses and cognitive activity in humans. Slow waves like Theta and Delta are usually associated with sub conscious mind, whereas alpha waves represent relaxed state of mind and are more prominent at occipital and parietal lobes. High frequency waves like Beta and Gamma waves represents active state of mind at frontal and other areas of the brain. As these frequency waves at certain scalp locations exhibit important cognitive behaviour. In this paper, we tried to extract cognitive features based on the specific EEG band and scalp location to analyse AUD and identifying correlation with IGD.

\section{RELATED WORK}

In this section, we shade light on the existing works. For the simplicity, this section is divided in 3-subsections to cover AUD, IAD and summary.

\section{A. Alcohol Use Disorder (AUD)}

There are many subjective questionnaires which were tweaked according to study to screen for alcohol misuse which are ranging from CAGE, the TWEAK, and AUDIT-C 
[13]. But the main issue is with the admittance of the subject. Even with the higher prevalence rate among other behavioural disorders, it is not diagnosed more than $30 \%$. Hence, it is desired that the questionnaire should be brief, highly sensitive, focused on consequences including demographic features. Even with those careful designed questionnaire, it is giving underfitting or overfitting results, which is demanding the objective approach to diagnose the AUD. From last decade researcher focused to include neurological modalities in their method and apparatus.

Study conducted in [14] has highlighted the challenges of questionnaire methods. QEEG [14] was used as neuroimaging modality and applied method on healthy controls and disordered subjects. EEG recording in setup came with different noise, which further removed to make noise-free EEG data. Later, features like absolute power (AP) and relative power (RP) were extract from preprocessed data. When machine learning methods were applied as classification methods, classification accuracy (96\%), sensitivity (97\%) and specificity (93\%) were achieved indicating efficacy of QEEG method to diagnose AUD.

Effect of alcoholism on different human organs were discussed in [15]. Authors [15] referred data which was collected for 122 subjects and 120 trials of each subjects using 64 channel EEG device. Features qualification for class discrimination were tested using Kruskal-Wallis statistical test and then fed into LS-SVM classifier with two different kernels and resulting in accuracy of $96.67 \%$ and $97.92 \%$ respectively. These results concluded that identification using brain waves of alcoholic subjects lead to better results as compared to other subjective or physical tests, reason being external changes are not a constrain for EEG signals.

Authors [16] conducted study on alcoholics and nonalcoholic subjects. Three-band orthogonal wavelet filter bank was used to extract standard signal bands after removal of noise signal from full band EEG. Logarithmic power was identified as one of the discriminating features, which was used to classify the test population. The data was mapped to higher dimensional space and used new optimal hyperplane to minimize classification error. Hence, achieved $97.08 \%$ classification accuracy.

\section{B. Internet Gaming Disorder (IGD)}

When prolong interface of excessive gaming, it shows symptoms like withdrawal symptoms, tolerance, not able to control playing time, unsuccessful quitting attempts, giving up other activities, continuing to play despite problems, playing more to relieve negative moods, risk having jeopardized social and personal relationship which depicts addictive behaviour. Here, we reviewed on IGD with different tools and methods.

To avoid the under rating and over rating, Tao et al [17] conducted a study where the method was designed to included clinically significant impairment criterion and course criterion along with internet addiction criterion. Cumulative diagnostic score was calculated considering weighted value of these criterion, which was able to distinguish addicted subjects from normal persons. The proposed diagnostic criteria was evaluated in three steps, developmental stage to yield the results on subjects, followed by cross validity of criterion inclusion and at last criteria were evaluated by multiple psychiatrist in order to determine inter-rater reliability.

Orsolya et al. [18] studied was to develop and validate brief psychometric instrument based of which they could justify usefulness and validity of each of the IGD symptoms as criteria. Item Response Theory (IRT) was used as method to design the task. For this study Facebook and a gamingrelated website, helped in getting gamer sample. To induce the craving and enhance participation the reward of 300 Euros were offered in form of lottery incentive. To test psychometric properties of IGD criteria first confirmatory factor analysis was done and then structural regression model were used on outcome result. Another parameter was cut-off threshold, investigated using Latent Class Analysis (LCA). During analysis 3 criteria's: (a) result validity; (b) reliability; and (c) suitability were suggested for future IGD's research.

Addiction-specific cues are somewhere linked to motivational relevance and reward processing. With the brain neurology and behavioural study, this idea came up by considering the assumption of specific brain property. It is noted that brain reacts to selective environmental stimuli not all simultaneously, which most of the time also related to the behavioural property of the observer.

Duven et al. [19] had presented a semi-natural EEG design. This research was developed under that environment subjects which had to interface with the computer game and play for specific ERPs. Here, EEG recordings were captured to assess reward processing. Peaks were identified which were used to detect differences between peaks of the N100, N200, P200, P300. In the result as hypothesis explained, high score was indicated for IGD subjects than the healthy control group. As a result, the authors [19] reached to the conclusion that IGD subjects indeed show a divergent processing of reward stimuli in the designed virtual environment. The data gathered during this study had showed the excessive behaviour effect, which are similar to the symptoms exhibited by subjects with substance use disorder. Further authors [19] had suggested more natural paradigm should be the part of IGD studies to understand behaviour complexity and complex nature of the IGD.

Two of the studies $[20,21]$ were performed to for the assessment on the brain reflective system in internet addiction for subjects to know who are computer game players. This study was done in resting state and different brain network region was the areas of interest specific focus was on "default mode network" and that region reflect nontask related cognitive processes. Beta band power recorded as reduced in first study, which was also observed in ADHD patients during task-related impulsivity. Along with this the Gamma waves power was also recorded that increases in absolute terms. It is noted that in other studies of impulsivity that gamma band power found changed. In resting state, gamma waves were found most associated with the impulsive symptom in online gaming addicts. It was suggested that it can be the biomarker for pathological understanding of IGD. On the other hand, second study 
findings indicated that decreased absolute delta and beta band powers are found affected in addicted subjects, which if further investigated on large population may be one of the biomarkers to classify the IGD patients even in comorbid state.

\section{Summary}

Despite of broader acceptability and use of questionnairebased assessment in researchers and clinicians, there is discordance with brain imaging reports found in some studies. Major percentage of researchers have used EEG as modality to understand brain regions involvement and activation during event related potentials (ERP). Modelling of EEG data is easy using different available EEG algorithm, which highlights the activation of region of interest in the result. Related works suggested that EEG as the method of assessment gives better result in locating specific functional changes in brain and resulting better accuracy. In EEG based studies, it has been found that AUD and IGD share most of the common symptoms like depression, anxiety, relapsing, affected reward processing. Our proposed method will check the associated information and extract most relevant information as feature. The outcome of the proposed method will be helpful in drawing the result to support the findings.

\section{Proposed Method And Simulation}

Here, MATLAB 2015 and EEGLab is used to process EEG signals. Figure 2 presents the block diagram of the proposed method. The method starts by accruing the EEG data. The EEG dataset used to perform experiments have been aacquired from the University of California Irvine knowledge discovery in database [22] (we have collected data from existing study performed by "Henri Begleiter" [22] in which genetic predisposition to alcoholism was examined to find EEG correlates. Other studies have also used this data to understand the alcohol subjects behaviour [24-27].), which was partially handled in respect of other artefacts epoch removal. Outliers have been removed from raw data, which reduced the overall size of data. Generally, EEG data are rich in feature. For the proposed method, we have focused on one of the spectral features which exhibited important brain state in case of addicted subjects and healthy controls.

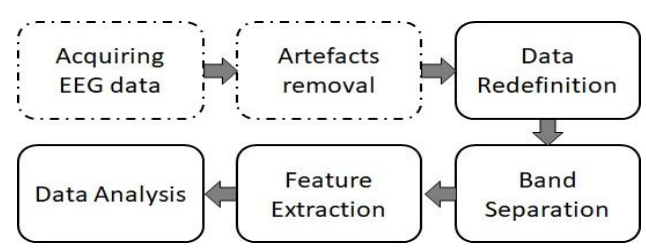

Fig. 1. Block diagram of proposed model for EEG data analysis.

\section{A. The Dataset and Preliminary Operations}

Alcoholic and control subject EEG dataset used under this study acquired from available EEG database [22]. This is one of common database which is used in many studies and originally collected by a longitudinal study on large number of subjects to correlated the EEG signals findings on alcoholic patients. Data recording have been performed on all montages according to international standard of 10-20 system. For each subject discrete trial of 120 numbers of each 1 second taken. The data has been recorded for eye open state with event related potentials of 90 images. 64 channel data have been recorded and stored for different subjects. Reference electrode has been placed over nose, which had impedance value less than $5 \mathrm{k} \mathrm{ohm}$. The dataset includes the EEG recordings acquired from entire 10/20 international montage or standard sites. Each of the subjects involved has undergone 120 trials for different stimuli consisting of 90 images. These EEG signals also contain event-related bio-potentials obtained by 64 montages. Ground electrode has been placed on nose with the impedance less than $5 \mathrm{k}$ ohm. As electrooculography (EOG) signal is considered as noise which has been removed through the reading deviation on 2 different electrodes that was occurred due to vertical and horizontal sudden eye movement. Sampling frequency $256 \mathrm{~Hz}$ has been set while data recording. The raw data was checked for outliers which later removed from original recorded dataset and the size of data also reduced.

\section{B. Data Conversion}

The collected data was not found suitable to process directly to EEGLab. First, we analyzed the data to understand the information field and converted into another EEG format. Raw data has multiple trials for different samples in different file, which is accumulated into single EEG structure for each sample and empty information which has been inferred from data source is also embed into structure to make it complete for further processing. Subject group information, stimuli information, sensor position and sample information have been managed in the EEG structure.

\section{Statistical Analysis}

We noted that band power has been found as one of the discriminating features. When we plotted the collected EEG data there is visible difference seen on overall band power. In order to understand the information contained within data we determined the band power in the form of power spectral density for all band and channel.

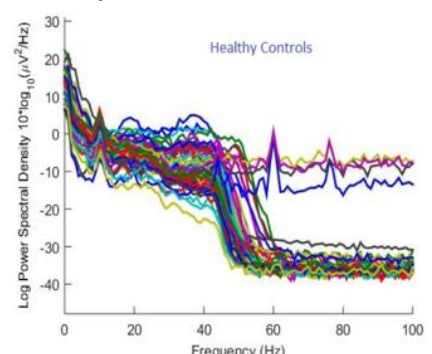

(a)

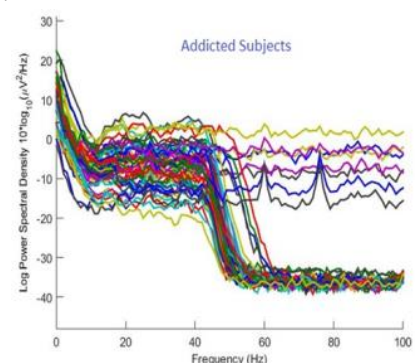

(b)
Fig. 3. Full Band EEG Signal. (a) For healthy controls; (b) For addicted subjects.

The raw EEG data which we have built during initial step contains high and low frequency signals along with EEG five bands which can be seen in the full band signal as shown in Figure 3.

Data has been passed through band pass and notch filter to keep the related bands only and to remove the artefacts like EOG, EMG, EEG and power line signal (Equation (1) is used to do this). 


$$
h_{d}[n]=\left\{\begin{array}{cc}
\frac{\sin \left(\omega_{c 2}(n-M)\right)}{\pi(n-M)}-\frac{\sin \left(\omega_{c 1}(n-M)\right)}{\pi(n-M)} & n \neq M \\
\frac{\omega_{c 2}-\omega_{c 1}}{\pi} & n=M
\end{array}\right.
$$

Filtered data has clearer picture about the power difference in AUD and normal subjects that can also be seen in Figure 4.

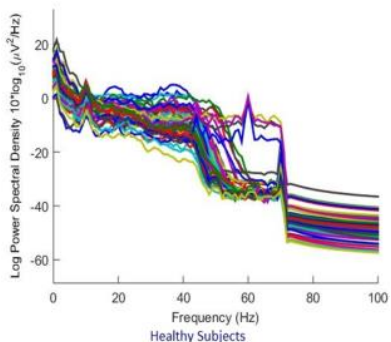

(a)

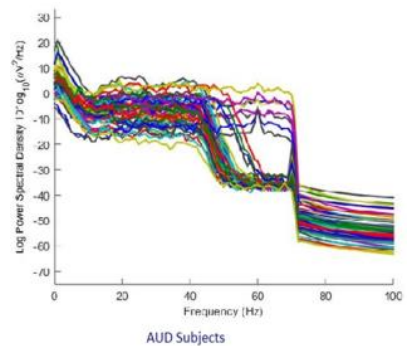

(b)
Fig. 4. Filtered EEG data. (a) For healthy subjects. (b) for AUD subjects

After pre-processing on data EEG bands are separated by applying Fast Fourier Transform (FFT) with the transformation equation (2).

$$
X(k)=\frac{1}{N} \sum_{n=0}^{N-1} x(n) e^{-j(2 k \pi n / N)} \quad k=0,1, \ldots, N-1
$$

The separated band power is then calculated using PSD function for all channels, which is visibly found different in addicted and normal subjects. Equation (3) - (7) is used to determine the channel band power respectively for alpha, beta, delta, theta and gamma channels.

$$
\begin{aligned}
\text { Alpha } & =\sum_{i=1}^{N}\left(S_{\text {xAlpha }}(i)\right)^{2} \\
\text { Beta } & =\sum_{i=1}^{N}\left(S_{\text {xBeta }}(i)\right)^{2} \\
\text { Delta } & =\sum_{i=1}^{N}\left(S_{\text {xDelta }}(i)\right)^{2} \\
\text { Theta } & =\sum_{i=1}^{N}\left(S_{\text {xTheta }}(i)\right)^{2} \\
\text { Gamma } & =\sum_{i=1}^{N}\left(S_{\text {xGamma }}(i)\right)^{2}
\end{aligned}
$$

To sketch the difference between AUD and healthy subjects, we evaluated the mean of PSD for subjects of each group and presented in Table I.

\section{RESULTS AND DISCUSSION}

For both groups (AUD and Normal), mean PSD is calculated and presented graphically in Figure (5) - (7). Figure (5) to (7) shows the channel wise PSD value. It can be utilized to present the power difference. No major difference in gamma power on central and parietal region is observed for the AUD and normal subjects, but Gamma waves are found increased in FP1 and FP2 channels of prefrontal region and $\mathrm{T} 7$ and $\mathrm{T} 8$ channels of temporal region of AUD subjects. Figure 5 highlights the power difference between two groups. Figure 6 indicates the difference in the power value of all channels for beta waves which are found decreased for F3, F4, Fz, F7 channels in frontal region and at $\mathrm{O} 1, \mathrm{O} 2$ channels at occipital region for addicted patients.
Someone experiencing and feeling deep and raw emotions, his mental state can be highlighted from Theta band of EEG recording. Theta band value found decreased for alcohol subjects for almost all channels, but significant decrease in frontal region at channels F3, F4, F7, F8, Fz and occipital region channels $\mathrm{O} 1, \mathrm{O} 2$.

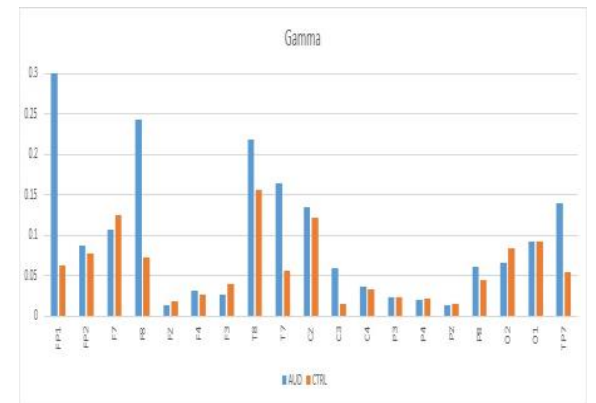

Fig. 5. Mean PSD value at gamma band

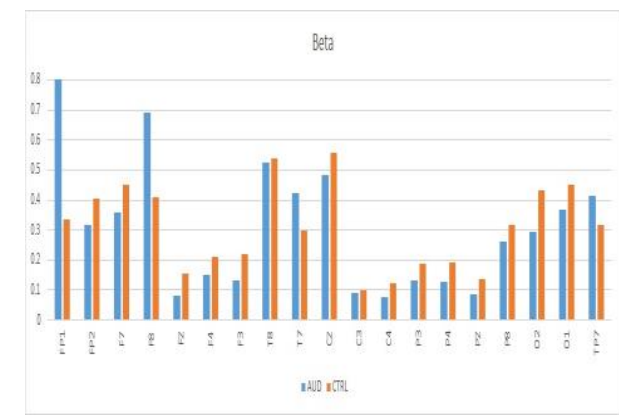

Fig. 6. Mean PSD value at beta band

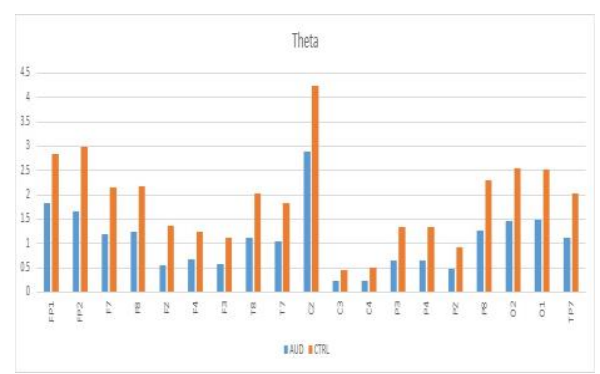

Fig. 7. Mean value of PSD at theta band

As optimal value of gamma wave indicate better information processing and learning in normal controls but high value in addicted subjects indicate high arousal and stress along with poor information processing capability. Beta waves at occipital regions are low indicating delayed response in identifying stimuli in addicted subjects. Theta waves decrease at frontal region, indicate imbalance in emotional expression of AUD subjects. As seen in the study conducted by Kim et al. [24] theta, beta and gamma waves also recorded similar difference in healthy and IGD subjects.

\section{CONCLUSIONS}

In this paper, we have proposed a novel method for analysing alcohol use disorder and define the ccorrelation with internet gaming disorder using human cognition. We have discussed the human brain structure and presented the relation with EEG signals in Section II which was further explored experimentally. Extensive experiments were conducted using benchmark dataset. We presented rigorous analysis by considering cognitive aspects of each frequency band and channel and reported the results in Table I. 
TABLE I

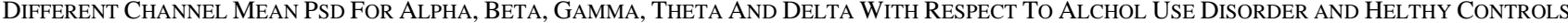

\begin{tabular}{|c|c|c|c|c|c|c|c|c|c|c|c|c|c|c|c|c|c|c|c|}
\hline \multirow{2}{*}{$\begin{array}{l}\text { Channels } \rightarrow \\
\text { Bands } \downarrow\end{array}$} & FP1 & FP2 & F7 & F8 & $\mathbf{F Z}$ & F4 & $\mathbf{F 3}$ & T8 & T7 & $\mathbf{C Z}$ & $\mathbf{C 3}$ & $\mathrm{C4}$ & P3 & P4 & $\mathbf{P Z}$ & P8 & $\mathbf{O 2}$ & 01 & TP7 \\
\hline & \multicolumn{19}{|c|}{ Alcohol Use Disorder Subjects } \\
\hline Alpha & 1.28 & 1.21 & 1.20 & 1.18 & 0.51 & 0.66 & 0.62 & 1.01 & 1.14 & 1.83 & 0.30 & 0.30 & 0.60 & 0.56 & 0.43 & 1.10 & 1.13 & 1.20 & 1.18 \\
\hline Beta & 1.61 & 0.32 & 0.36 & 0.69 & 0.08 & 0.15 & 0.13 & 0.53 & 0.42 & 0.48 & 0.09 & 0.08 & 0.13 & 0.13 & 0.09 & 0.26 & 0.29 & 0.37 & 0.42 \\
\hline Gamma & 15.33 & 0.09 & 0.11 & 0.24 & 0.01 & 0.03 & 0.03 & 0.22 & 0.17 & 0.13 & 0.06 & 0.04 & 0.02 & 0.02 & 0.01 & 0.06 & 0.07 & 0.09 & 0.14 \\
\hline Theta & 1.84 & 1.67 & 1.19 & 1.24 & 0.55 & 0.66 & 0.57 & 1.12 & 1.04 & 2.88 & 0.24 & 0.23 & 0.66 & 0.65 & 0.47 & 1.26 & 1.45 & 1.50 & 1.12 \\
\hline \multirow[t]{2}{*}{ Delta } & 16.07 & 14.70 & 9.92 & 9.20 & 2.86 & 4.46 & 3.70 & 5.76 & 5.41 & 25.54 & 1.44 & 1.22 & 3.08 & 3.13 & 2.56 & 6.15 & 7.00 & 6.43 & 4.89 \\
\hline & \multicolumn{19}{|c|}{ Healthy Controls } \\
\hline Alpha & 1.36 & 1.37 & 1.47 & 1.48 & 0.70 & 0.81 & 0.82 & 1.55 & 1.38 & 1.97 & 0.47 & 0.57 & 0.89 & 1.00 & 0.65 & 1.55 & 1.66 & 1.62 & 1.36 \\
\hline Beta & 0.33 & 0.41 & 0.45 & 0.41 & 0.15 & 0.21 & 0.22 & 0.54 & 0.30 & 0.56 & 0.10 & 0.12 & 0.19 & 0.19 & 0.14 & 0.31 & 0.43 & 0.45 & 0.32 \\
\hline Gamma & 0.06 & 0.08 & 0.12 & 0.07 & 0.02 & 0.03 & 0.04 & 0.16 & 0.06 & 0.12 & 0.02 & 0.03 & 0.02 & 0.02 & 0.02 & 0.04 & 0.08 & 0.09 & 0.06 \\
\hline Theta & 2.85 & 3.00 & 2.14 & 2.18 & 1.36 & 1.24 & 1.11 & 2.02 & 1.82 & 4.23 & 0.45 & 0.49 & 1.33 & 1.33 & 0.92 & 2.30 & 2.53 & 2.52 & 2.02 \\
\hline Delta & 23.08 & 23.72 & 13.09 & 13.87 & 6.95 & 6.82 & 6.58 & 8.50 & 8.16 & 33.21 & 1.82 & 1.93 & 6.09 & 5.82 & 4.20 & 10.62 & 11.01 & 10.93 & 8.14 \\
\hline
\end{tabular}

We noted that theta and beta bands mean absolute power has been reduced in the frontal and prefrontal brain region. Reduction in beta power in prefrontal region amounts leads to slow response inhibition and compulsive behaviour. Kim et al [24] focused on frontal and parietal region to find the severity of IGD and they found symptoms are matching with impulsive behaviour. In addition, we found similarities in terms of emotional imbalance, high arousal, slow response inhibition from EEG data analysis. These results concluded that AUD and IGD are correlated. As comorbidity is the norm, more than one behavioral and neurological disorder can coexist. In future, EEG based study on other addiction can also be considered to find the correlation with IGD.

\section{REFERENCES}

[1] Hannah Ritchie and Max Roser (2019) - "Alcohol consumption". Published online at OurWorldInData.org. Retrieved from: 'https://ourworldindata.org/alcohol-consumption' [Online Resource]

[2] Feigin, V.L., Abajobir, A.A., Abajobir, A.A.a., 2017. Global, regional, and national burden of neurological disorders during 19902015: a systematic analysis for the global burden of disease study 2015. The Lancet. Neurology 16, 877-897. doi:10.1016/S14744422(17) 30299-5.

[3] Kuss, D. J., \& Griffiths, M. D. (2012). Internet gaming addiction: A systematic review of empirical research. International Journal of Mental Health and Addiction, 10(2), 278-296.

[4] Kapur M, Tuteja A, Prasad S, Bawankule N (2017) Online gaming in india: Reaching a new pinnacle. https://assets.kpmg/content/dam/kpmg/in/pdf/2017/05/onlinegaming.pdf

[5] Razieh J., Ali G., Zaman A., Narjesskhatoon S. The relationship between Internet addiction and anxiety in the universities' students. Interdisciplinary Journal of Contemporary Research in Business. 2012;4:942-949

[6] Pontes, H. M., Patrão, I. M., \& Griffiths, M. D. (2014). Portuguese validation of the Internet Addiction Test: An empirical study. Journal of behavioral addictions, 3(2), 107-14

[7] Schivinski, B., Brzozowska-Woś, M., Buchanan, E. M., Griffiths, M. D., \& Pontes, H. M. (2018). Psychometric assessment of the Internet Gaming Disorder diagnostic criteria: An Item Response Theory study. Addictive behaviors reports, 8, 176-184. doi:10.1016/j.abrep.2018.06.004

[8] Chakraborty K, Basu D, Kumar K. Internet addiction: Consensus, controversies, and the way ahead. East Asian Archives of Psychiatry. 2010 Sep;20(3):123-32.

[9] Peukert P, Sieslack S, Barth G, Batra A. Internet- and computer game addiction: Phenomenology, comorbidity, etiology, diagnostics and therapeutic implications for the addictives and their relatives. Psychiatrische Praxis. 2010 Jul;37(5 ):219-24.

[10] Martin CS, Chung T, Kirisci L, Langenbucher JW. Item response theory analysis of diagnostic criteria for alcohol and cannabis use disorders in adolescents: Implications for DSM-5. J Abnorm Psychol 2006;115:807-14

[11] Jonathan G. Westman, Spencer Bujarski, Lara A. Ray, Impulsivity Moderates Subjective Responses to Alcohol in Alcohol-Dependent Individuals, Alcohol and Alcoholism, Volume 52, Issue 2, March 2017, Pages 249-255, https://doi.org/10.1093/alcalc/agw096
[12] Wetherill, R. R., \& Fromme, K. (2009). Subjective responses to alcohol prime event-specific alcohol consumption and predict blackouts and hangover. Journal of studies on alcohol and drugs, 70(4), 593-600.

[13] Fagbemi, Keji. (2011). Q: What is the best questionnaire to screen for alcohol use disorder in an office practice?. Cleveland Clinic journal of medicine. 78. 649-51. 10.3949/ccjm.78a.10186.

[14] Mumtaz, Wajid \& Vuong, Pham \& Xia, Likun \& Malik, Aamir \& Abd Rashid, Rusdi. (2016). Automatic Diagnosis of Alcohol Use Disorder using EEG Features. Knowledge-Based Systems. 105. 10.1016/j.knosys.2016.04.026

[15] priya, anchala \& Yadav, Pooja \& Jain, Shweta \& Bajaj, Varun. (2018). An efficient method for classification of alcoholic and normal EEG signals using EMD. The Journal of Engineering. 2018. 10.1049/joe.2017.0878

[16] Sharma, Manish \& Deb, Dipankar \& Acharya, U Rajendra. (2017). A novel three-band orthogonal wavelet filter bank method for an automated identification of alcoholic EEG signals. Applied Intelligence. 10.1007/s10489-017-1042-9.

[17] Tao R, Huang X, Wang J, Zhang H, Zhang Y, Li M. Proposed diagnostic criteria for Internet addiction. Addiction 2010;105:556-64.

[18] Orsolya Király, Pawel Sleczka, Halley M. Pontes, Róbert Urbán, Mark D. Griffiths, Zsolt Demetrovics, Validation of the Ten Item Internet Gaming Disorder Test (IGDT-10) and evaluation of the nine DSM-5 Internet Gaming Disorder criteria, Addictive Behaviors, Volume 64, 2017, Pages 253-260, ISSN 0306-4603, doi:10.1016/j.addbeh.2015.11.005.

[19] Duven E, Mller K, Beutel M, Wlfling K (2014) Altered reward processing in pathological computer gamers erp-results from a seminatural gamingdesign. Brain and Behavior 5, DOI 10.1002/brb3.293

[20] Andrews-Hanna, J.R., Reidler, J.S., Huang, C., \& Buckner, R.L. (2010). Evidence for the default network's role in spontaneous cognition. Journal of Neurophysiology, 104, 322-335.

[21] Greicius, M.D., \& Menon, V. (2004). Default-mode activity during a passive sensory task: uncoupled from deactivation but impacting activation. Journal of Cognitive Neuroscience, 16, 1484-1492

[22] Begleiter Henri, Ingber Lester (1999) Examining EEG correlates of genetic predisposition to alcoholism. https://archive.ics.uci.edu/ml/datasets/eeg+database

[23] Kim, Minah \& Lee, Takhyung \& Choi, Jung-Seok \& Kwak, Yoo Bin \& Hwang, Wu Jeong \& Kim, Taekwan \& Yoon Lee, Ji \& Lim, Jae-A \& Park, Minkyung \& Jin Kim, Yeon \& Nyun Kim, Sung \& Kim, DaiJin \& Soo Kwon, Jun. (2017). Neurophysiological correlates of altered response inhibition in internet gaming disorder and obsessivecompulsive disorder: Perspectives from impulsivity and compulsivity. Scientific Reports. 7. 41742. 10.1038/srep41742.

[24] X.L. Zhang, H. Begleiter, B. Porjesz, W. Wang, and A. Litke. (1995). "Event related potentials during object recognition tasks". Brain Research Bulletin. Volume 38. Number 6. Pages 531-538.

[25] L. Ingber. (1997). Statistical mechanics of neocortical interactions: Canonical momenta indicators of electroencephalography. Physical Review E. Volume 55. Number 4. Pages 4578-4593.

[26] L. Ingber. (1998). Statistical mechanics of neocortical interactions: Training and testing canonical momenta indicators of EEG. Mathematical Computer Modelling. Volume 27. Number 3. Pages 3364.

[27] J. G. Snodgrss and M. Vanderwart. (1980). "A standardized set of 260 pictures: norms for the naming agreement, familiarity, and visual complexity." Journal of Experimental Psychology: Human Learning and Memory. Volume 6. Pages 174-215.

[28] Margaret A. Boden. Mind as machine: A history of cognitive science. Oxford University Press, 2008. 